\title{
Topology optimization of viscoelastic rectifiers
}

\author{
Jensen, Kristian Ejlebjærg; Szabo, Peter; Okkels, Fridolin
}

Published in:

Applied Physics Letters

Link to article, DOI:

$10.1063 / 1.4728108$

Publication date:

2012

Document Version

Publisher's PDF, also known as Version of record

Link back to DTU Orbit

Citation (APA):

Jensen, K. E., Szabo, P., \& Okkels, F. (2012). Topology optimization of viscoelastic rectifiers. Applied Physics Letters, 100(23), Paper 234102. https://doi.org/10.1063/1.4728108

\section{General rights}

Copyright and moral rights for the publications made accessible in the public portal are retained by the authors and/or other copyright owners and it is a condition of accessing publications that users recognise and abide by the legal requirements associated with these rights.

- Users may download and print one copy of any publication from the public portal for the purpose of private study or research.

- You may not further distribute the material or use it for any profit-making activity or commercial gain

- You may freely distribute the URL identifying the publication in the public portal

If you believe that this document breaches copyright please contact us providing details, and we will remove access to the work immediately and investigate your claim 


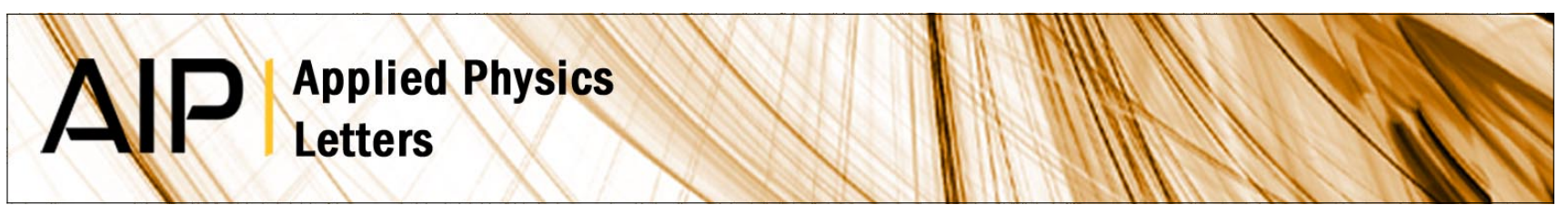

\section{Topology optimization of viscoelastic rectifiers}

Kristian Ejlebjerg Jensen, Peter Szabo, and Fridolin Okkels

Citation: Appl. Phys. Lett. 100, 234102 (2012); doi: 10.1063/1.4728108

View online: http://dx.doi.org/10.1063/1.4728108

View Table of Contents: http://apl.aip.org/resource/1/APPLAB/v100/i23

Published by the American Institute of Physics.

\section{Related Articles}

Real-time digital compensation to reduce acceleration's sensitivity in quartz resonator

Rev. Sci. Instrum. 83, 064706 (2012)

A method to integrate patterned electrospun fibers with microfluidic systems to generate complex microenvironments for cell culture applications

Biomicrofluidics 6, 024131 (2012)

DNA capture-probe based separation of double-stranded polymerase chain reaction amplification products in poly(dimethylsiloxane) microfluidic channels

Biomicrofluidics 6, 026503 (2012)

Optical actuation of microelectromechanical systems using photoelectrowetting Appl. Phys. Lett. 100, 224103 (2012)

Nanometer-size anisotropy of injection-molded polymer micro-cantilever arrays

J. Appl. Phys. 111, 103530 (2012)

\section{Additional information on Appl. Phys. Lett.}

Journal Homepage: http://apl.aip.org/

Journal Information: http://apl.aip.org/about/about_the_journal

Top downloads: http://apl.aip.org/features/most_downloaded

Information for Authors: http://apl.aip.org/authors

\section{ADVERTISEMENT}

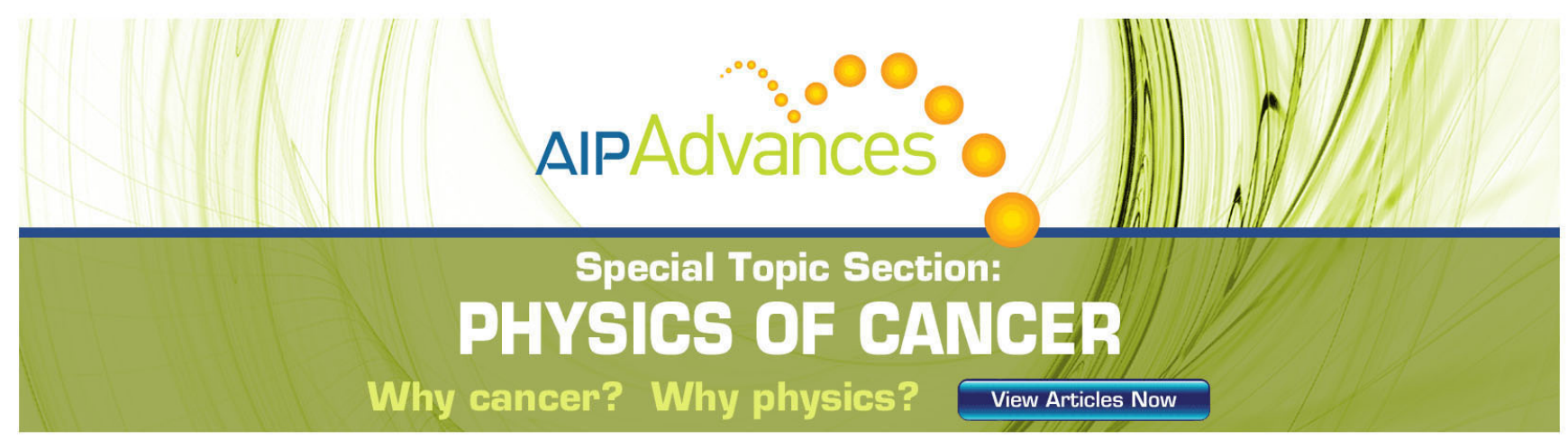




\title{
Topology optimization of viscoelastic rectifiers
}

\author{
Kristian Ejlebjerg Jensen, ${ }^{1, a)}$ Peter Szabo, ${ }^{2}$ and Fridolin Okkels ${ }^{1}$ \\ ${ }^{1}$ Department of Micro- and Nanotechnology, Technical University of Denmark, DK-2800 Kgs. Lyngby, \\ Denmark \\ ${ }^{2}$ Department of Chemical and Biochemical Engineering, Technical University of Denmark, DK-2800 Kgs. \\ Lyngby, Denmark
}

(Received 2 November 2011; accepted 18 May 2012; published online 8 June 2012)

\begin{abstract}
An approach for the design of microfluidic viscoelastic rectifiers is presented based on a combination of a viscoelastic model and the method of topology optimization. This presumption free approach yields a material layout topologically different from experimentally realized rectifiers, and simulations indicate superior performance for the optimized design in the regime of moderate elasticity. (C) 2012 American Institute of Physics. [http://dx.doi.org/10.1063/1.4728108]
\end{abstract}

Micropumps are needed for medical delivery as well as lab-on-a-chip systems, and they can be constructed using a variety of actuation principles. ${ }^{1}$ Interest has gathered around the possibility of combining an oscillating diaphragm with no-moving-parts valves/rectifiers, which rely on the inertial properties of the fluid for their working mechanism. ${ }^{2}$ In example, the method of topology optimization has been applied to reproduce the Tesla valve as the optimal design for these devices. ${ }^{3}$ Inertial effects, and thus also the performance of inertial rectifiers, however decreases as devices are scaled down. Viscoelastic effects on the other hand do not vanish at the micro scale, and therefore rectifiers have been suggested ${ }^{4,5}$ on this basis.

Topology optimization with a memory free nonNewtonian fluid has been demonstrated, ${ }^{6}$ but the working mechanism of viscoelastic rectifiers is related solely to the memory of the fluid due to past deformations. Topology optimization considering fluid memory has not previously been demonstrated, and this is probably due to the fact that merely modeling such a fluid in complex geometries has been a long standing challenge for the scientific community. We find that it is possible to combine recent model developments ${ }^{7}$ with a high level implementation of topology optimization ${ }^{8}$ to determine the optimal material layout that maximizes the flow rate ratio in a rectifier device. The optimization allows for porous material, so to confirm that the design does not rely on this, we perform simulations without it, and such a quantitative investigation of viscoelastic rectifiers outside an experimental setting has not previously been demonstrated.

The fluid memory is described with a differential constitutive model, where the spatial configuration of, e.g., the molecules, or whatever gives rise to the viscoelastic properties, is taken into account. A popular approach is to study a solution of spring connected point mass pairs (dumbbells) in a Newtonian solvent considering only orientation and elongation of fluid elements. In such models, the conformation tensor $\mathbf{A}$ is used to describe configurations, and it is related to the dumbbell end-to-end vector a, such that $\operatorname{Trace}(\underline{\underline{\mathbf{A}}})=\left\langle\mathbf{a}^{2}\right\rangle / \mathrm{a}_{\mathrm{eq}}^{2}$, where $\langle\cdots\rangle$ is a statistical average, and $\mathrm{a}_{\mathrm{eq}}$ is the equilibrium length of the end-to-end vector. The finite extensibility model by Chilcott and Rallison ${ }^{9}$ fea-

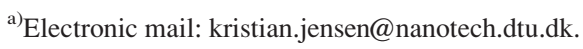

tures both a finite maximum dumbbzell extensibility $\mathrm{a}_{\max }$ as well as a constant shear viscosity, like Boger fluids ${ }^{10}$

$$
\begin{aligned}
-\frac{k(\underline{\underline{\mathbf{A}}})}{\lambda}(\underline{\underline{\mathbf{A}}}-\underline{\underline{\mathbf{I}}}) & =\frac{D \underline{\underline{\mathbf{A}}}}{D t}-\left[\underline{\underline{\mathbf{A}}} \cdot \nabla \mathbf{v}+(\nabla \mathbf{v})^{T} \cdot \underline{\underline{\mathbf{A}}}\right], \\
k(\underline{\underline{\mathbf{A}}}) & =\frac{1}{1-\operatorname{Trace}(\underline{\underline{\mathbf{A}}}) / \mathbf{a}_{\max }^{2}}, \\
\underline{=}_{e} & =\frac{\eta_{p}}{\lambda} k(\underline{\underline{\mathbf{A}}})(\underline{\underline{\mathbf{A}}}-\underline{\underline{\mathbf{I}}}),
\end{aligned}
$$

where $\mathbf{I}$ is the identity matrix, $\mathbf{v}$ is the velocity, $\lambda$ is the dumbbell relaxation time, $D / D t$ is the material derivative, $\eta_{p}$ is a dumbbell viscosity, and $k(\underline{\underline{\mathbf{A}}})$ can be thought of as a nonlinear spring constant modification. $\tau$ is the dumbbell stress tensor, which is put into the Stokes equation, when assuming a creeping isothermal and incompressible viscoelastic fluid. Adding the usual continuity equation for mass conservation yields

$$
\begin{gathered}
\mathbf{0}=\boldsymbol{\nabla} \cdot\left(-p \underline{\underline{\mathbf{I}}}+\eta_{s} \underset{\underline{\dot{\gamma}}}{=}+\underset{=}{=}\right), \\
0=\boldsymbol{\nabla} \cdot \mathbf{v},
\end{gathered}
$$

where $p$ is the pressure, $\eta_{s}$ is the solvent viscosity, and $\underset{\underline{\gamma}}{\dot{\gamma}}$ $=\boldsymbol{\nabla} \mathbf{v}+(\boldsymbol{\nabla} \mathbf{v})^{T}$ is the rate of deformation tensor. It has been shown that the solution of Eqs. (1)-(5) with the finite element method is troubled by the existence of discontinuous velocity gradients $\boldsymbol{\nabla} \mathbf{v}$, at element borders. ${ }^{11}$ The remedy is to construct a continuous approximation $\underline{\underline{\mathbf{G}}}$, for use on the right hand side of Eq. (1) and to add $\eta_{p}\left(\underline{\underline{\dot{\gamma}}}-\underline{\underline{\mathbf{G}}}-\underline{\underline{\mathbf{G}}}^{T}\right)$ as a zero on the right hand side of the Stokes Eq. (4). A significant development occurred with the introduction of the logconformation $\operatorname{method}^{7}$ involving a substitution $\underline{\underline{\mathbf{A}}}=e^{\underline{\underline{\mathbf{s}}}}$, such that Eq. (1) is transformed to take the form

$$
\frac{D \stackrel{=}{\underline{\mathbf{s}}}}{D t}=\underline{\underline{\mathbf{R}}}(\underline{\underline{\mathbf{s}}}, \underline{\underline{\mathbf{G}}}),
$$

where the computation of the reaction term $\mathbf{R}$ as well as the conformation tensor $e^{=}$involves calculation of eigenvectors and eigenvalues of $\mathbf{s}^{7,12}$ This change of variables guarantees 
the positive definiteness of the conformation tensor, making it a much more robust formulation. It however also complicates the equations and associated linearizations significantly, and therefore, the optimization in this work relies heavily on a commercial high level finite element package ${ }^{13}$ and related implementation of topology optimization. ${ }^{8}$

We implement topology optimization by adding the usual Darcy damping term ${ }^{14}-\alpha(\rho) \mathbf{v}$, to the right hand side of the Stokes equation (4). The idea is that wherever the design variable $\rho$ is equal to unity, the damping term vanishes such that the governing equation for a fluid domain is recovered; conversely, where the design variable is equal to zero, it results in very large damping terms $\alpha_{\max }$, such that the velocity becomes marginal, and the no slip boundary condition is enforced in an approximative way. A continuous optimization problem can then be formulated by interpolating the damping term in the design variable, but the convergence properties of the optimization is sensitive to the choice of interpolation. In this work, a PDE filter ${ }^{15}$ is applied to the design variable producing a filtered design variable $\tilde{\rho}$, with a minimum length scale $L_{\min }$, Eq. (6). Then, a projection function, ${ }^{16} \mathrm{Eq}$. (7), defines the projected design variable $\bar{\rho}$, which is used in the usual convex relation, ${ }^{14} \mathrm{Eq}$. (8).

$$
\begin{gathered}
\tilde{\rho}=\rho+L_{\min }^{2} \nabla^{2} \tilde{\rho}, \\
\bar{\rho}=\frac{1}{2}+\frac{\tanh \left(\xi\left(\tilde{\rho}-\frac{1}{2}\right)\right)}{2 \tanh (\xi / 2)}, \\
\alpha=\alpha_{\max } \frac{q(1-\bar{\rho})}{\bar{\rho}+q} .
\end{gathered}
$$

Here $\xi$ defines the steepness of the projection function, while the convexity of the damping term in the projected design variable is determined by $q$.

Introducing a characteristic length scale $L$, pressure $\Delta p^{*}$, and damping $\alpha_{\max }$ allows for the governing equations to be written in dimensionless form ${ }^{17}$ such that the following dimensionless parameters arise

$$
\mathrm{Da}=\frac{\eta_{s}+\eta_{p}}{L^{2} \alpha_{\max }}, \quad \beta=\frac{\eta_{s}}{\eta_{s}+\eta_{p}}, \quad \text { and } \quad \mathrm{We}=\lambda \frac{\Delta p^{*}}{\eta_{s}+\eta_{p}} .
$$

The Darcy number Da, describes the magnitude of the viscous term relative to the damping term in solid regions. Hence, excessively large Da will give a bad approximation of the no slip boundary condition, while convergence problems will arise for the optimization with too small Da. $\beta$ expresses the proportion of viscous effects due to the solvent, while the Weissenberg number We indicates the relative strength of elastic to viscous effects. Experimental rectifiers work most efficiently in the regime of high elasticity/We, where a transition to unsteady flow occurs, whereas we focus on optimization of steady solutions in the regime of moderate elasticity and correspondingly smaller driving pressures and/or relaxation times. Note that the Weissenberg number and thus also the device performance is independent of the characteristic length scale provided the driving pressure is fixed, and inertia can be neglected.

We use a standard approach ${ }^{12}$ for both the stabilization of the convective equation and for the representation of the various physical variables. The filtered design variable is represented by second order Lagrange elements, while the design variable itself is considered constant in all elements. Furthermore, an isotropic triangular mesh is used to avoid favored design directions.

We use a fully implicit scheme to evolve in time for 20 dumbbell relaxation times starting from a viscoelastic fluid at rest, and then proceed by initializing a non-linear solver with the final transient solution. ${ }^{18}$ Optimization iterations without steady solutions can occur, in which case the last transient solution is used for the sensitivity analysis. Although this approach is inconsistent, it does not become an issue for the optimization, since unsteady flows is a rare occurrence at the Weissenberg numbers considered. Our optimization setup is periodic and pressure driven as sketched in Fig. 1. The objective function $\phi$, to be minimized is the flow rate ratio

$$
\phi=\frac{\dot{V}_{\leftarrow}}{\dot{V}_{\rightarrow}}=\frac{\int-\mathbf{v}_{\leftarrow} \cdot \hat{\mathbf{x}} d \mathbf{r}}{\int \mathbf{v}_{\rightarrow} \cdot \hat{\mathbf{x}} d \mathbf{r}},
$$

where the arrows indicate the flow configuration. We compute the objective function gradient $\partial \phi / \partial \rho$, with an adjoint method and combine it with the method of moving asymptotes ${ }^{19}$ for updating the design variables.

In terms of model parameters we choose $\beta=0.59$, as it is representative of Boger fluids and used widely in benchmarks of numerical algorithms for this reason. To avoid early transition to unsteady flows $\mathrm{a}_{\max }^{2}=100$ can be used, and with this we are able to perform optimizations at $\mathrm{We}=5$ without serious issues with unsteady solutions. The driving pressure is set at $7.17 \Delta p^{*}$ to give a unity average velocity for the initial empty design and thus also an effective We number closer to the imposed. Finally, we find that the optimization performs well with a characteristic mesh size $h=L / 10, L_{\min }=h, \mathrm{Da}=10^{-5}, q=4 \cdot 10^{-6}$, and $\xi=10$.

Optimizations with and without imposed symmetry both result in a contraction followed by an obstacle as shown ${ }^{17}$ in Figs. 2(a) and 2(b). The working mechanism is best understood by considering Figs. 2(c) $-2(\mathrm{~g})$ specific to the symmetric case, while adopting the dumbbell fluid picture and focusing on the strongly accelerating nature of the flow in the obstacle wake close to the rear stagnation point: The acceleration will cause the forward dumbbell mass to move

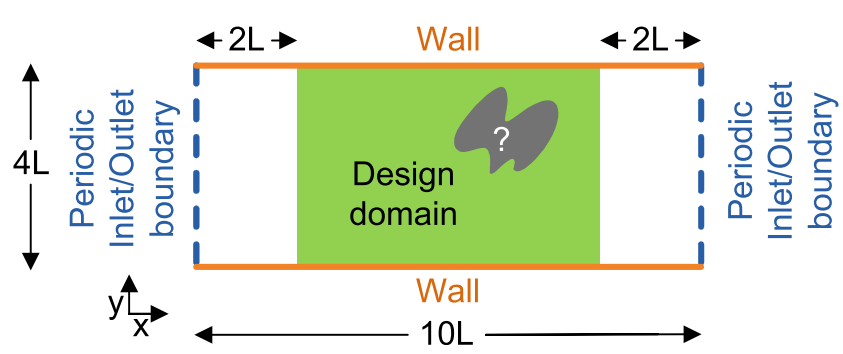

FIG. 1. Optimization setup with periodic velocity vector and conformation tensor at the inlet/outlet boundaries, where the pressure is fixed at either 0 or $\Delta p$ depending on the flow configuration $(\rightarrow$ or $\leftarrow$ ). There is no tangential stress at the inlet/outlet boundaries and only pressure contributes to the normal stress. The no slip boundary condition is imposed on the top and bottom boundaries, and the design variable is defined in the central rectangle only. 
(a)

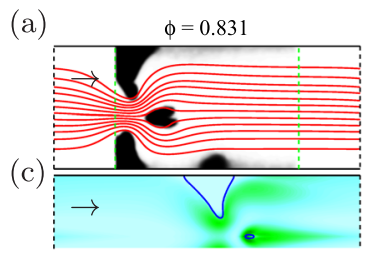

(b)

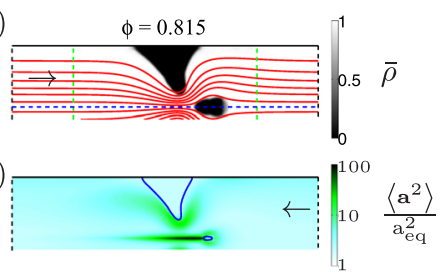

(e)
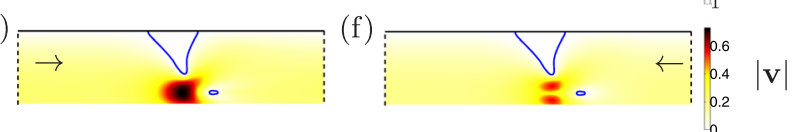

(g)

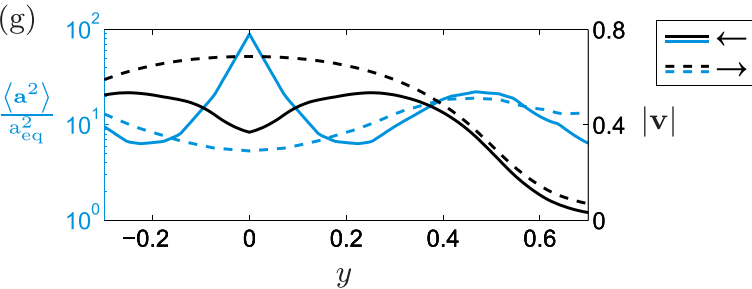

FIG. 2. The filtered design variable is plotted together with streamlines for optimizations without (a) and with (b) symmetry. Both the dumbbell extension (c)-(d) and the velocity magnitude (e)-(f) are shown in the symmetric case for the two flow directions together with a $6 \cdot 10^{-4}$ contour of the projected design variable in blue. The working mechanism is illustrated by plotting the dumbbell extension and velocity magnitude through a cross section connecting the contractions (g).

faster than the rear mass, and in this way, a wake of elongated dumbbells appears, Figs. 2(c) and 2(d). The dumbbells are particularly elongated in the reverse flow configuration due to the high flow velocity in the contraction, and the wake causes a damping that gives rise to a local velocity minimum in the contraction center, Figs. 2(e)-2(g).

As indicated in Fig. 2, the effective size of the obstacle is smaller than a plot of the filtered design variable on a linear scale suggests. This is due to the relation between the filtered design variable and the damping term (Eq. (8)), and it means that the projected design variable should be thresholded around $6 \cdot 10^{-4}$ to produce a performing design without porous material. It however seems that it is the curvature in the left region of the obstacle that is essential, which allows for the use of a significantly larger airfoil like obstacle.

The results of symmetric simulations with a state-of-theart hyperbolic design ${ }^{5}$ and a design derived from topology optimization are shown ${ }^{17}$ in Fig. 3 in the case of boundary conditions and model parameters identical to that of the topology optimization. Although unsteady flows are often encountered, the objective function shows clear convergence up to $\mathrm{We}=5$ with respect to spatial discretization and simulation time (not shown). The simulations indicate that the

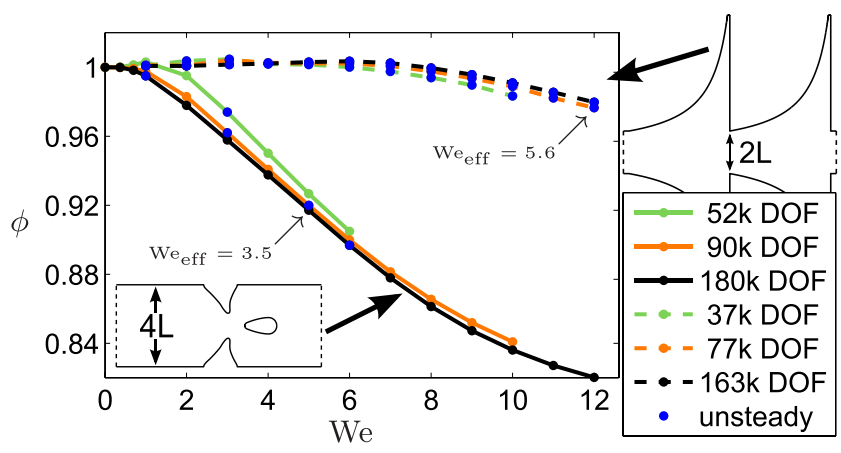

FIG. 3. Two designs are characterized in terms of their flow rate ratio as a function of the Weissenberg number for different number of degrees of freedom (DOF). Effective Weissenberg numbers $\mathrm{We}_{\mathrm{eff}}$ are calculated as $2 \mathrm{WeV} \dot{V}_{\rightarrow} / L_{\text {cont }}^{2}$, where $L_{\text {cont }}$ is the width of the contractions.

contraction-obstacle-design not only has superior performance in the considered regime but also that the rectification effect sets in strongly at small Weissenberg numbers.

In conclusion, we have presented results for topology optimization of a viscoelastic rectifier and found a design that promises superior performance in the regime of moderate elasticity.

${ }^{1}$ B. Iverson and S. Garimella, Microfluidics Nanofluidics 5, 145 (2008).

${ }^{2}$ E. Stemme and G. Stemme, Sens. Actuators A 39, 159 (1993).

${ }^{3}$ G. Pingen and K. Maute, Comput. Math. Appl. 59, 2340 (2010).

${ }^{4}$ A. Groisman and S. Quake, Phys. Rev. Lett. 92, 94501 (2004).

${ }^{5}$ P. Sousa, F. Pinho, M. Oliveira, and M. Alves, J. Non-Newtonian Fluid Mech. 165, 652 (2010).

${ }^{6}$ G. Pingen, A. Evgrafov, and K. Maute, Int. J. Computat. Fluid Dyn. 22, 457 (2008).

${ }^{7}$ M. Hulsen, R. Fattal, and R. Kupferman, J. Non-Newtonian Fluid Mech. 127, 27 (2005).

${ }^{8}$ L. Olesen, F. Okkels, and H. Bruus, Int. J. Numer. Methods Eng. 65, 975 (2006).

${ }^{9}$ M. Chilcott and J. Rallison, J. Non-Newtonian Fluid Mech. 29, 381 (1988).

${ }^{10}$ D. Boger, J. Non-Newtonian Fluid Mech. 3, 87 (1977).

${ }^{11}$ A. Fortin, R. Guenétte, and R. Pierre, Comput. Methods Appl. Mech. Eng. 189, 121 (2000).

${ }^{12}$ L. Carracciuolo, D. Casaburi, L. D'Amore, G. D'Avino, P. Maffettone, and A. Murli, J. Non-Newtonian Fluid Mech. 166, 1382 (2011).

${ }^{13}$ COMSOL 3.5a Multiphysics Reference Guide (2008).

${ }^{14}$ T. Borrvall and J. Petersson, Int. J. Numer. Methods Fluids 41, 77 (2003).

${ }^{15}$ B. Lazarov and O. Sigmund, Int. J. Numer. Methods Eng. 86, 765 (2011).

${ }^{16}$ F. Wang, B. Lazarov, and O. Sigmund, Struct. Multidiscip. Optim. 43, 767 (2011).

${ }^{17}$ See supplementary material at http://dx.doi.org/10.1063/1.4728108 for non-dimensional equations, implementation validation plot and additional simulation figures.

${ }^{18}$ The implementation uses default parameters, ${ }^{13}$ and it has been verified against a Ref. 7 in a benchmark geometry. ${ }^{17}$

${ }^{19}$ K. Svanberg, Int. J. Numer. Methods Eng. 24, 359 (1987). 